\title{
ON LINEAR SINGULAR FUNCTIONAL-DIFFERENTIAL EQUATIONS IN ONE FUNCTIONAL SPACE
}

\author{
ANDREI SHINDIAPIN
}

Received 1 October 2002

We use a special space of integrable functions for studying the Cauchy problem for linear functional-differential equations with nonintegrable singularities. We use the ideas developed by Azbelev and his students (1995). We show that by choosing the function $\psi$ generating the space, one can guarantee resolubility and certain behavior of the solution near the point of singularity.

\section{Linear Volterra operators in $\Delta_{\psi}$ spaces}

We consider the following $n$-dimensional functional-differential equation:

$$
\mathscr{L} x \stackrel{\text { def }}{=} \dot{x}+(K+S) \dot{x}+A x(0)=f,
$$

where

$$
\begin{gathered}
(K y)(t)=\int_{0}^{t} K(t, s) y(s) d s, \\
(S y)(t)= \begin{cases}B(t) y[g(t)] & \text { if } g(t) \in[0,1], \\
0 & \text { if } g(t) \notin[0,1] .\end{cases}
\end{gathered}
$$

The case where $K$ and $S$ are continuous on $L_{p}[0,1]$ operators is well studied (see, e.g., [1] and the references therein). Here we suppose that the functions $K(t, s)$ and $B(t)$ may be nonintegrable at $t=0$. More precisely, we will formulate conditions on operators $K$ and $S$ in Sections 2 and 3. Under such conditions, those operators are not bounded on $L[0,1]$ and one has to choose other functional spaces for studying (1.1). We propose a space of integrable functions on $[0,1]$ and show that it may be useful in such a case.

We call $\Delta_{\psi}^{p}$ space the space of all measurable functions $y:[0,1] \rightarrow \mathbb{R}^{n}$, for which

$$
\|y\|_{\Delta_{\psi}^{p}}=\sup _{0<h \leq 1} \frac{1}{\psi(h)}\left(\int_{0}^{h}|y(s)|^{p} d s\right)^{1 / p}<\infty .
$$


We assume everywhere below that $\psi$ is a nondecreasing, absolutely continuous function, $\psi(0)=0$.

Theorem 1.1. The space $\Delta_{\psi}^{p}$ is a Banach space.

Let $X[a, b], Y[a, b]$ be spaces of functions defined on $[a, b]$.

We will call $V: X[0,1] \rightarrow Y[0,1]$ the Volterra operator [3] if for every $\xi \in[0,1]$ and for any $x_{1}, x_{2} \in X[0,1]$ such that $x_{1}(t)=x_{2}(t)$ on $[0, \xi],\left(V x_{1}\right)(t)=\left(V x_{2}\right)(t)$ for $t \in[0,1]$.

It is possible to say that each Volterra operator $V: X[0,1] \rightarrow Y[0,1]$ generates a set of operators $V_{\xi}: X[0, \xi] \rightarrow Y[0, \xi]$, where $\xi \in(0,1]$. By $y_{\xi}$, we denote the restriction of function $y$ defined on $[0,1]$ onto segment $[0, \xi]$.

Theorem 1.2. Let $V: L \rightarrow L$ be a linear bounded operator. Then $V$ is a linear bounded operator in $\Delta_{\psi}^{p}$ and $\|V\|_{\Delta_{\psi}^{p}} \leq\|V\|_{L^{p} \text {. }}$

Proof. Let $y \in \Delta_{\psi}^{p}$. Then

$$
\begin{aligned}
\|V y\|_{\Delta_{\psi}^{p}} & =\sup _{0<h \leq 1} \frac{1}{\psi(h)}\left\|\left(V_{\xi} y_{\xi}\right)\right\|_{L[0, \xi]^{p}} \\
& \leq \sup _{0<h \leq 1} \frac{1}{\psi(h)}\left\|V_{\xi}\right\|_{L[0, \xi]}\left\|y_{\xi}\right\|_{L[0, \xi]} \leq\|V\|_{L}\|y\|_{L^{p}} .
\end{aligned}
$$

Theorem 1.3. Let $V: \Delta_{\psi_{1}}^{p} \rightarrow \Delta_{\psi_{1}}^{p}$ be linear bounded operator and let

$$
\sup _{t \in[0,1]} \frac{\psi_{2}(t)}{\psi_{1}(t)}<\infty .
$$

Then $V$ is linear and bounded in $\Delta_{\psi_{2}}^{p}$ and

$$
\|V\|_{\Delta_{\psi_{2}}^{p}} \leq\|V\|_{\Delta_{\psi_{1}}^{p}} \sup _{\xi \in[0,1]} \sup _{\tau \in[0, \xi]} \frac{\psi_{1}(\xi) \psi_{2}(\tau)}{\psi_{2}(\xi) \psi_{1}(\tau)} .
$$

Proof. Let $y \in \Delta_{\psi 2}$. Then

$$
\begin{aligned}
\|V y\|_{\Delta_{\psi_{2}}^{p}} & \leq \sup _{\xi \in[0,1]} \frac{\|V y \xi\|_{L[0, \xi]} \psi_{1}(\xi)}{\psi_{2}(\xi) \psi_{1}(\xi)} \leq \sup _{\xi \in[0,1]} \frac{\left\|V y_{\xi}\right\|_{\Delta_{\psi_{1}}^{p}[0, \xi]} \psi_{1}(\xi)}{\psi_{2}(\xi)} \\
& \leq\|V\|_{M \psi_{1}}{ }^{p} \sup \frac{\left\|y_{\psi}\right\|_{\Delta_{\psi_{1}}^{p}} \psi_{1}(\xi)}{\psi_{2}(\xi)} \\
& \leq\|V\|_{M} \psi_{1} p \sup _{\xi \in[0,1]} \sup _{\tau \in[0, \psi]} \frac{\left\|y_{\tau}\right\|_{L[0, \tau]} \psi_{1}(\xi) \psi_{2}(\tau)}{\psi_{1}(\tau) \psi_{2}(\xi) \psi_{2}(\tau)} \\
& \leq\|y\|_{\Delta_{\psi_{2}}^{p}}\|V\|_{\Delta_{\psi 1}^{p}} \sup _{\xi \in[0,1]} \sup _{\tau \in[0, \xi]} \frac{\psi_{1}(\xi) \psi_{2}(\tau)}{\psi_{2}(\xi) \psi_{1}(\tau)} .
\end{aligned}
$$

Corollary 1.4. If $V_{1}: \Delta_{\psi_{1}}^{p} \rightarrow \Delta_{\psi_{1}}^{p}$ and $V_{2}: \Delta_{\psi_{2}}^{p} \rightarrow \Delta_{\psi_{2}}^{p}$ are linear continuous Volterra operators, then $V=V_{1}+V_{2}$ is continuous on space $\Delta_{\psi}^{p}$ generated by $\psi(t)=\min \left(\psi_{1}(t), \psi_{2}(t)\right)$ and $\|V\|_{\Delta_{\psi}^{p}} \leq\left\|V_{1}\right\|_{\Delta_{\psi_{1}}^{p}}+\left\|V_{2}\right\|_{\Delta_{\psi_{2}}^{p}}$. 


\section{Operator $K$}

In this section, we consider the integral operator (1.2). We will show that under certain conditions on matrix $K(t, s)$, a function $\psi$ may be indicated such that $K$ is bounded on $\Delta_{\psi}$ and its norm is limited by a given number.

We say that matrix $K(t, s)$ satisfies the $\mathcal{N}$ condition if for some $p$ and $p_{1}$ such that $1 \leq p \leq p_{1}<\infty$ and for any $\varepsilon \in(0,1]$,

$$
\left\|K_{\mathcal{\varepsilon}}(t, \cdot)\right\|_{L[0, t]} \in L_{p^{\prime}}[\varepsilon, 1]
$$

Here $K_{\varepsilon}(t, s)$ is a restriction of $K(t, s)$ onto $[\varepsilon, 1] \times[0, t], 1 / p+1 / p^{\prime}=1$.

The $\mathcal{N}$ condition admits a nonintegrable singularity at point $t=0$.

Lemma 2.1. Let nonnegative function $\omega:[0,1] \rightarrow \mathbb{R}$ be nonincreasing and having a nonintegrable singularity at $t=0$.

Then $\psi(t)=\exp \left[\int_{1}^{t} \omega(s) d s\right]$ is absolutely continuous on $[0,1]$, does not decrease, and is a solution of the equation $\int_{1}^{t} \omega(s) x(s) d s=x(t)$.

Denote

$$
\psi(t)=\exp \left[\frac{1}{C} \int_{1}^{t} \operatorname{vraisup}_{s \in[0, \tau]}\|K(\tau, s)\| d \tau\right] .
$$

Theorem 2.2. Let matrix $K(t, s)$ satisfy the $\mathcal{N}$ condition with $p=1$ and let $C$ be some positive constant. Then operator $K$ is bounded in $\Delta_{\psi}$ with function $\psi$ defined by the equality (2.2) and $\|K\|_{\Delta_{\psi}} \leq C$.

Proof. Let $x \in \Delta_{\psi}$ and $y=K x$. From the $\mathcal{N}$ condition it follows that for almost all $t \in$ $[0,1], K(\cdot, s) \in L_{\infty}$. Let $\omega(t)=\operatorname{vraisup}_{s \in[0, \tau]}\|K(\tau, s)\| d \tau$. Then

$$
\begin{aligned}
\left(\int_{0}^{t}\|y(s)\| d s\right) & \leq\left[\int_{0}^{t}\left(\int_{0}^{\tau}\|K(\tau, s)\|\|x(s)\| d s\right) d \tau\right] \\
& \leq \int_{0}^{t}(\underset{s \in[0, \tau]}{\operatorname{vraisup}}\|K(\tau, s)\|)\left(\int_{0}^{\tau}\|x(s)\| d s\right) d \tau \\
& \leq\|x\|_{\Delta_{\psi}} \int_{0}^{t} \omega(\tau) \psi(\tau) d \tau
\end{aligned}
$$

According to Lemma 2.1, $\psi(t)=\exp \left[(1 / C) \int_{1}^{t} \omega(s) d s\right]$ is a solution of the equation $\int_{1}^{t} \omega(s) \psi(s) d s=C \psi(t)$, does not decrease, is absolutely continuous, and $\psi(0)=0$. That implies

$$
\left(\int_{0}^{t}\|y(s)\| d s\right) \leq C\|x\|_{\Delta_{\psi}} \psi(t) .
$$


Remark 2.3. If $K(\cdot, s)$ has bounded variation on $s$, it is possible to indicate a "wider" space $\Delta_{\psi}$ for which conditions of Theorem 2.2 are satisfied by defining function $\psi$ as

$$
\psi(t)=\exp \left[\frac{1}{C} \int_{1}^{t}\left(\|K(\tau, \tau)\| d \tau+\int_{0}^{\tau} d_{s} \underset{s \in[0, \tau]}{\operatorname{var}}\|K(\tau, s)\|\right) d \tau\right] .
$$

Theorem 2.4. Let matrix $K(t, s)$ satisfy the $\mathcal{N}$ condition with $1<p<\infty$ and let $C$ be some positive constant. Then operator $K$ is bounded in space $\Delta_{\psi}^{p}$ generated by

$$
\psi(t)=\exp \left[\frac{1}{p C} \int_{1}^{t}\left(\int_{0}^{\tau}\|K(\tau, s)\|^{p^{\prime}} d s\right)^{p_{1} / p^{\prime}} d \tau\right]
$$

and $\|K\|_{\Delta_{\psi}^{p}} \leq C$.

Theorem 2.4 can be proved in a way similar to proof of Theorem 2.2.

Lemma 2.5. Let $K: \Delta_{\psi}^{p} \rightarrow \Delta_{\psi}^{p}(1<p<\infty)$ be a bounded operator and let its matrix $K(t, s)$ satisfy the $\mathcal{N}$ condition. Then $K: \Delta_{\psi}^{p} \rightarrow L_{p}$ is a compact operator.

Proof. For every $t \in[0,1],(K y)(t)$ is a linear bounded functional on $L_{p}$. Let $\left\{y_{i}\right\}$ be a sequence weakly converging to $y_{0}$ in $L_{p}$. If $\left\{y_{i}\right\} \subset \Delta_{\psi}^{p}$ and $\left\|y_{i}\right\|_{\Delta_{\psi}^{p}} \leq 1$, then $\left\|y_{0}\right\|_{\Delta_{\psi}^{p}} \leq 1$. Indeed, if for some $t_{1} \in[0,1],\left(\left(1 / \psi\left(t_{1}\right)\right) \int_{0}^{t_{1}}\|y(s)\| p d s\right)^{1 / p}>1$, then the sequence $l y_{i}=$ $\int_{0}^{1} l(s) y_{i}(s) d s$ does not converge to $l y_{0}$, where

$$
l(s)= \begin{cases}1, & \text { if } s \leq t_{1} \\ 0, & \text { if } s>t_{1}\end{cases}
$$

Hence, for almost all $t \in[0,1],\left\{\left(K y_{i}\right)(t)\right\}$ converges and the set $K y$ is compact in measure. Thus, for the operator $K: \Delta_{\psi}^{p} \rightarrow L_{p}$ to be compact, it is necessary and sufficient that the norms of $K y$ are equicontinuous for $\|y\|_{\Delta_{\psi}^{p}} \leq M$. Let $\delta \in(0,1)$. As $K: \Delta_{\psi}^{p} \rightarrow \Delta_{\psi}^{p}$ is a bounded operator,

$$
\left(\frac{1}{\psi(\delta)} \int_{0}^{\delta}\|(K y)(s)\|^{p} d s\right)^{1 / p} \leq \Delta_{0}
$$

This implies that for any $\varepsilon>0$, there exists $\delta_{1}>0$ such that if $\delta<\delta_{1}$, then $\left(\int_{0}^{\delta}\|(K y)(s)\|^{p} d s\right)^{1 / p} \leq \varepsilon / 2$.

Then, from the $\mathcal{N}$ condition, there exists $\delta_{2}$ such that if mese $\leq \delta_{2}$ for some $e \subset[\delta, 1]$, then $\left(\int_{e}\|(K y)(s)\|^{p} d s\right)^{1 / p} \leq \varepsilon / 2$.

Finally, for $e_{1} \subset[\delta, 1]$ such that mes $e_{1} \leq \min \left\{\delta_{1}, \delta_{2}\right\}$,

$$
\left(\int_{e_{1}}\|(K y)(s)\|^{p} d s\right)^{1 / p} \leq\left(\int_{0}^{\delta}\|(K y)(s)\|^{p} d s\right)^{1 / p}+\left(\int_{\delta}^{1}\|(K y)(s)\|^{p} d s\right)^{1 / p} \leq \varepsilon .
$$

Lemma 2.6. Let $\left\{y_{i}\right\} \rightarrow y_{0}$ in $L_{p}(1<p<\infty)$ and let the sequence $\left\{(1 / u) y_{i}\right\}$ be bounded in $\Delta_{\psi}^{p}$ for some continuous increasing function $u, u(0)=0$. Then $\left\{y_{i}\right\} \rightarrow y_{0}$ in $\Delta_{\psi}^{p}$. 
Proof. We have

$$
\left(\int_{0}^{t}\left\|y_{i}(s)\right\|^{p} d s\right)^{1 / p} \leq u(t)\left(\int_{0}^{t}\left\|\frac{y_{i}(s)}{u(s)}\right\|^{p} d s\right)^{1 / p} \leq M u(t) \psi(t) .
$$

Thus, $y_{i} \in \Delta_{\psi}^{p}$. Beginning with some $N$ for any $t \in[0,1]$ and for any given $\varepsilon>0$,

$$
\left(\int_{0}^{t}\left\|y_{i}(s)-y_{0}(s)\right\|^{p} d s\right)^{1 / p} \leq \varepsilon
$$

Hence,

$$
\begin{aligned}
\left(\int_{0}^{t}\left\|y_{0}(s)\right\|^{p} d s\right)^{1 / p} & \leq\left(\int_{0}^{t}\left\|y_{i}(s)-y_{0}(s)\right\|^{p} d s\right)^{1 / p}+\left(\int_{0}^{t}\left\|y_{i}(s)\right\|^{p} d s\right)^{1 / p} \\
& \leq \varepsilon+M u(t) \psi(t) \leq M u(t) \psi(t), \\
\left(\int_{0}^{t}\left\|y_{i}(s)-y_{0}(s)\right\|^{p} d s\right)^{1 / p} & \leq 2 M u(t) \psi(t),
\end{aligned}
$$

beginning with some $N_{\delta}$ for any $\delta>0,\left\|y_{0}-y_{i}\right\|_{\Delta_{\psi}^{p}}<\delta$. Indeed, Lemma 2.5 guarantees the existence of $\tau \in(0,1]$ such that for all $t \in[0, \tau]$,

$$
\left(\int_{0}^{t}\left\|y_{i}(s)-y_{0}(s)\right\|^{p} d s\right)^{1 / p} \leq \delta \psi(t)
$$

Let $t \in[\tau, 1]$. Then for $\varepsilon=\delta \psi(\tau),(2.11)$ yields (2.13) for all $t \in[0,1]$.

Let $u:[0,1] \rightarrow \mathbb{R}$ be a continuous increasing function, $u(0)=0$. Denote

$$
\psi(t)=\exp \left[\int_{1}^{t} \frac{1}{u(\tau)}\left(\int_{0}^{\tau}\|K(\tau, s)\|^{p^{\prime}} d s\right)^{p / p^{\prime}} d \tau\right] .
$$

Lemmas 2.5 and 2.6 imply the following theorem.

Theorem 2.7. Let matrix $K(t, s)$ satisfy the $\mathcal{N}$ condition with $1<p<\infty$. And let $\psi$ be defined by (2.14). Then $K: \Delta_{\psi}^{p} \rightarrow \Delta_{\psi}^{p}$ is a compact operator and its spectral radius is equal to zero.

\section{Operator $S$}

Denote

$$
\begin{aligned}
& \left(S_{g} y\right)(t)= \begin{cases}y[g(t)] & \text { if } g(t) \in[0,1], \\
0 & \text { if } g(t) \notin[0,1],\end{cases} \\
& (S y)(t)=B(t)\left(S_{g}\right)(t) .
\end{aligned}
$$

In [2], it is shown that $S_{g}$ is bounded in $L_{p}$ if $r=\left(\sup \left(\operatorname{mes} g^{-1}(E) / \operatorname{mes} E\right)\right)^{1 / p}<\infty$ and $\left\|S_{g}\right\|_{L_{p}}=r$, where sup is taken on all measurable sets from $[0,1]$. 
Let $\Omega_{m}$ be a set of points from $[0,1]$ for which $g(t) \geq m t, \beta(t)$ is a nonincreasing majorant of function $\|B(t)\|$, and

$$
\varphi(t)=\lim _{\text {mese } \rightarrow 0} \frac{\operatorname{mes} g^{-1}(e)}{\text { mese }},
$$

where $e$ is a closed interval containing $t$.

We say that operator $S_{g}$ satisfies the $M$ condition if $\operatorname{vraisup}_{t \in[\varepsilon, 1]} \varphi(t)<\infty$ for any

$$
\varepsilon \in(0,1] \underset{t \in[\varepsilon, 1]}{\operatorname{vraisup}}\|B(t)\|<\infty,
$$

and there exists $m \in[0,1)$ such that

$$
\mu_{m}=\underset{t \in g\left(\Omega_{m}\right)}{\operatorname{vraisup}}\left(\beta(t)^{p} \varphi(t)\right)<\infty .
$$

Lemma 3.1. There exists nonincreasing function $u:(0,1] \rightarrow \mathbb{R}$ such that $\beta(t)^{p} \varphi(t) \leq u(t)$ and the function

$$
\psi(t)= \begin{cases}t^{u(t)} & \text { if } t \in(0,1] \\ 0 & \text { if } t=0,\end{cases}
$$

is absolutely continuous on $[0,1]$.

Proof. Let $\left\{t_{i}\right\}$ be a decreasing sequence, $t_{1}=1, t_{i} \rightarrow 0$. Denote

$$
n_{i}=\operatorname{vraisup}_{t \in\left(t_{i+1}, t_{i}\right)}\left(\beta(t)^{p} \varphi(t)\right), \quad u(t)=\frac{n_{i+1}-n_{i}}{t_{i+1}-t_{i}}\left(t-t_{i}\right)+n_{i},
$$

where $t \in\left(t_{i+1}, t_{i}\right)$. Then $\beta(t)^{p} \varphi(t) \leq u(t), u$ increases and is absolutely continuous on $[0,1]$.

Let

$$
v_{m}=m^{u(1)}\left[u(1)-\frac{1}{\ln m}\right]
$$

Theorem 3.2. Let operator $S_{g}$ satisfy the $M$ condition and let function u satisfy conditions of Lemma 3.1. Then $S_{g}$ is bonded in $\Delta_{\psi}^{p}$ with $\psi(t)=t^{u(t)}$ and

$$
\left\|S_{g}\right\|_{\Delta_{\psi}^{p}} \leq\left(\nu_{m}+\mu_{m}\right)^{1 / p} .
$$

Proof. Let $y \in \Delta_{\psi}^{p},\|y\|_{\Delta_{\psi}^{p}}=1$, and $\delta \in(0,1)$. Denote measures $\lambda$ and $\mu$ on $[\delta, 1]$ by $\lambda(e)=$ $\int_{e} \beta(s)^{p} d s$ and $\mu(e)=\int_{g^{-1}(e)} \beta(s)^{p} d s$. Then by the Radon-Nikodym [2] theorem, we have

$$
\begin{aligned}
\left\|\int_{\delta}^{t}\left|\left(S_{g} y\right)(t)\right|^{p} d s\right\| & \leq \int_{g^{-1}([0, t]) \cap[\delta, 1]}\|y[g(s)]\|^{p} d \lambda(s) \\
& =\int_{g^{-1}([0, t]) \cap[\delta, 1]}\|y(s)\|^{p} \frac{d \mu}{d \lambda}(s) d \lambda(s) .
\end{aligned}
$$


Then as $g(t) \leq t$,

$$
\frac{d \mu}{d \lambda}(s)=\lim _{\text {mese } \rightarrow 0} \frac{\int_{g^{-1}(e)} \beta(s)^{p} d s}{\int_{e} \beta(s)^{P} d s} \leq \lim _{\text {mese } \rightarrow 0} \frac{\operatorname{vraisup}_{g^{-1}(e)} \beta(s)^{p} d s}{\operatorname{vraisup}_{e} \beta(s)^{p}} \varphi(s)=\varphi(s)
$$

or

$$
\begin{aligned}
\left\|\int_{\delta}^{t}\left|\left(S_{g} y\right)(t)\right|^{p} d s\right\| \leq & \int_{g^{-1}\left([0, t] \backslash \Omega_{m}\right) \cap[\delta, 1]} \beta(s)^{p}\|y(s)\|^{p} \varphi(s) d s \\
& +\int_{g^{-1}\left(\Omega_{m}\right) \cap[\delta, 1]} \beta(s)^{p}\|y(s)\|^{p} \varphi(s) d s \\
\leq & \int_{0}^{m t} \beta(s)^{p}\|y(s)\|^{p} \varphi(s) d s+\int_{0}^{t}\|y(s)\|^{p} \mu_{m} d s \\
\leq & \int_{0}^{m t}\|y(s)\|^{p} u(s) d s+\mu_{m} \psi(t)^{p} .
\end{aligned}
$$

We denote function $u_{k}:(0,1] \rightarrow \mathbb{R}$ by $u_{k}(t)=u\left(t_{i}\right)$, where $t_{i}=\left(2^{k}-i\right) / 2^{k}, i=0,1,2, \ldots$, $2^{k}-1$. From $u_{k} \rightarrow u$, it follows that

$$
\int_{0}^{m t}\|y(s)\|^{p} u(s) d s=\lim _{k \rightarrow 0} \int_{0}^{m t}\|y(s)\|^{p} u_{k}(s) d s .
$$

We write function $u_{k}$ in the form

$$
u_{k}(t)=\left\{\begin{array}{lc}
u\left(t_{0}\right), & \text { if } t \in\left(t_{1}, t_{0}\right] \\
u\left(t_{0}\right)+\left[u\left(t_{1}\right)-u\left(t_{0}\right)\right], & \text { if } t \in\left(t_{2}, t_{1}\right] \\
\vdots & \vdots \\
u\left(t_{k-2}\right)+\left[u\left(t_{k-1}\right)-u\left(t_{k-2}\right)\right], & \text { if } t \in\left(t_{k}, t_{k-1}\right] .
\end{array}\right.
$$

The condition $t<t_{i}$ implies that $\int_{0}^{m t}\|y(s)\|^{p} d s \leq \psi^{p}(m t)=(m t)^{u(m t)} \leq m^{p u\left(t_{i}\right)} \psi^{p}(t)$ and

$$
\begin{aligned}
\int_{0}^{m t}\|y(s)\|^{p} u(s) d s & \leq \sum_{i=1}^{2^{k}} m^{p u\left(t_{i}\right)}\left[u\left(t_{i}\right)-u\left(t_{i-1}\right)\right] \psi^{p}(t)+u(1) m^{p u(1)} \psi^{p}(t) \\
& \leq \psi^{p}(t)\left[\int_{u(1)}^{\infty} m^{s} d s+m^{u(1)} u(1)\right] \\
& \leq \psi^{p}(t) m^{u(1)}\left[u(1)-\frac{1}{\ln m}\right]
\end{aligned}
$$

simultaneously for all $k$. Finally,

$$
\begin{aligned}
\left\|\int_{0}^{t}\left|\left(S_{g} y\right)(s)\right|^{p} d s\right\| & =\lim _{\delta \rightarrow 0}\left\|\int_{\delta}^{t}\left|\left(S_{g} y\right)(s)\right|^{p} d s\right\| \\
& \leq \psi^{p}(t) m^{u(1)}\left[u(1)-\frac{1}{\ln m}\right]+\psi^{p}(t) \mu_{m} \\
& \leq \psi^{p}(t)\left(\nu_{m}+\mu_{m}\right)
\end{aligned}
$$

which proves the theorem. 
Remark 3.3. From (3.7) and (3.8), it follows that if $\lim _{m \rightarrow 1}<1$, then there exists function $\psi$ such that the norm of operator $S_{g}: \Delta_{\psi}^{p} \rightarrow \Delta_{\psi}^{p}$ is less than 1 .

In some particular cases, it is possible to give less strict conditions on function $\psi$ generating the space $\Delta_{\psi}^{p}$. Direct calculations prove the following theorem.

Theorem 3.4. Let $B(t) \leq C_{1} / t^{\alpha}$ and $g(t)=C_{2} t^{\beta}$ with $\beta>1$. Then $\left\|S_{g}\right\|_{\Delta_{\psi}^{p}} \leq C_{1} / C_{2}$, where $\psi(t)=t^{\gamma}, \gamma \geq(\alpha p+\beta-1) / p(\beta-1)$. If $\gamma>(\alpha p+\beta-1) / p(\beta-1)$, then the spectral radius of $S_{g}$ is equal to zero.

\section{The Cauchy problem}

We consider the Cauchy problem for (1.1):

$$
(\mathscr{L} x)(t)=f(t), \quad x(0)=\alpha
$$

The theorems of this section are immediate corollaries of Theorems 2.2, 2.4, 2.7, 3.2, and 3.4.

Theorem 4.1. Let matrix $K(t, s)$ satisfy the $\mathcal{N}$ condition and let operator $S_{g}$ satisfy the $M$ condition. Let also vraisup $\operatorname{se}_{t \in[0,1]} u(t)=\infty,\left(\mu_{m}\right)^{1 / p} \leq q<1$, and let the function $\psi_{1}$ be given by (2.14). Then if $C<1-q$, the Cauchy problem (4.1) has a unique solution in $\Delta_{\psi}^{p}$ with $\psi(t)=\min \left\{\psi_{1}(t), t^{u(t)}\right\}$ for $f$ and $\alpha$ such that $(f-\alpha A) \in \Delta_{\psi}^{p}$.

Let $\omega$ be a solution of the equation

$$
m^{\omega}\left(\omega-\frac{1}{\ln m}\right) \leq C_{1}^{p}-q, \quad \gamma=\sup _{t \in[0,1]}\{u(t), \omega\}
$$

where $0 \leq q \leq C_{1}^{p}<1$, and $u$ satisfies conditions of Lemma 3.1.

Theorem 4.2. Let matrix $K(t, s)$ and operator $S_{g}$ satisfy the $\mathcal{N}$ and $M$ conditions, respectively. Let $\operatorname{vraisup}_{t \in[0,1]} u(t)<\infty$ and $\left(\mu_{m}\right)^{1 / p} \leq q<1$. Then if $q<C_{1},\left(C_{1}+C_{2}\right)<1$, then the Cauchy problem (4.1) has a unique solution in $\Delta_{\psi}^{p}$ with $\psi(t)=\min \left\{\psi_{1}(t), t^{\gamma}\right\}$ for $f$ and $\alpha$ such that $(f-\alpha A) \in \Delta_{\psi}^{p}$.

Theorem 4.3. Let matrix $K(t, s)$ satisfy the $\mathcal{N}$ condition, $B(t) \leq C_{1} / t^{\alpha}, g(t)=C_{2} t^{\beta}(\beta>1)$, and $\gamma>(\alpha p+\beta-1) / p(\beta-1)$. Let also $C<1$ and $\psi(t)=\min \left\{\psi_{1}(t), t^{\gamma}\right\}$. Then the Cauchy problem (4.1) has a unique solution for $f$ and $\alpha$ such that $(f-\alpha A) \in \Delta_{\psi}^{p}$.

Example 4.4. The Cauchy problem

$$
\begin{gathered}
\dot{x}(t)+p(t) \frac{x[h(t)]}{t^{k}}+q(t) \dot{x}\left(t^{2}\right)=f(t), \quad t \in[0,1], \\
x(\xi)=0, \quad \text { if } h(\xi) \leq 0,
\end{gathered}
$$

where $h(t) \leq t, k>1$, and $p$ and $q$ are bounded functions, has a solution if $\int_{0}^{t}|f(s)| d s \leq$ $M \exp \left(-t^{1-k}\right)$. If $(t-h(t)) \geq \tau>0$, then it has a solution if $\int_{0}^{t}|f(s)| d s \leq M t^{\gamma}$ for $\gamma>1$. 


\section{References}

[1] N. V. Azbelev, V. Maksimov, and L. Rakhmatullina, Introduction to the Theory of Linear Functional-Differential Equations, Advanced Series in Mathematical Science and Engineering, vol. 3, World Federation Publishers Company, Georgia, 1995.

[2] N. Dunford and J. T. Schwartz, Linear Operators. I. General Theory, Pure and Applied Mathematics, vol. 7, Interscience Publishers, New York, 1958.

[3] A. N. Tikhonov, On functional Volterra type equations and their applications to problems of mathematical physics, Moscow University Bulletin, Section A 1 (1938), no. 8, 1-25.

Andrei Shindiapin: Department of Mathematics and Informatics, Eduardo Mondlane University, 257 Maputo, Mozambique

E-mail address: andrei@sm.luth.se 


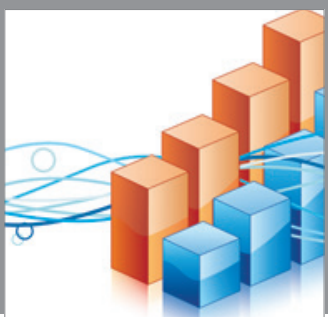

Advances in

Operations Research

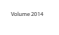

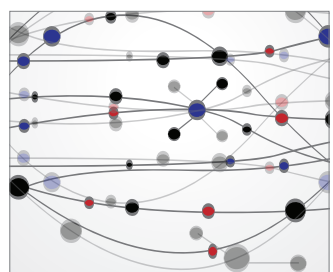

\section{The Scientific} World Journal
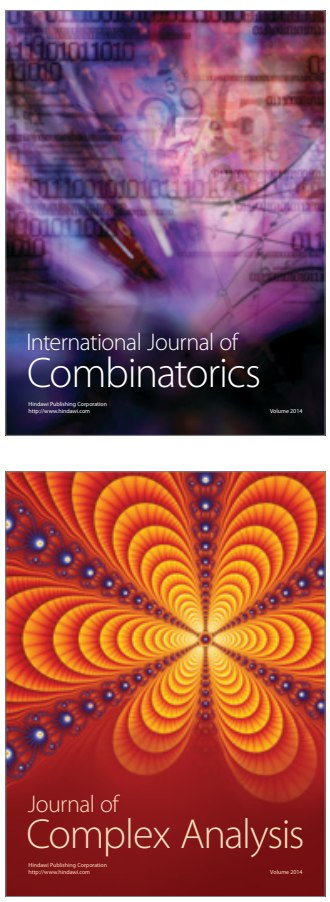

International Journal of

Mathematics and

Mathematical

Sciences
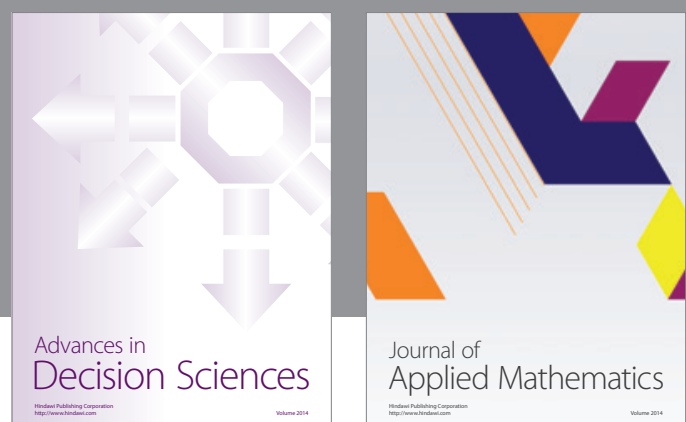

Journal of

Applied Mathematics
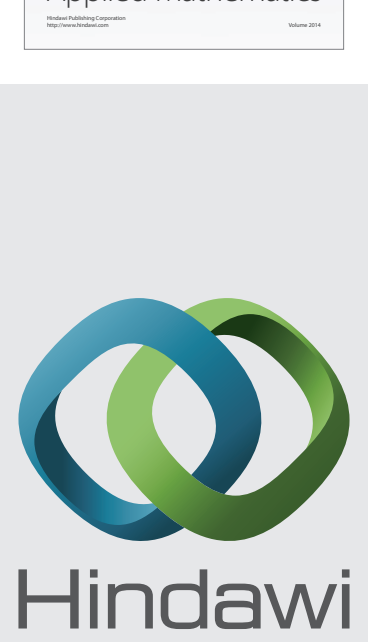

Submit your manuscripts at http://www.hindawi.com
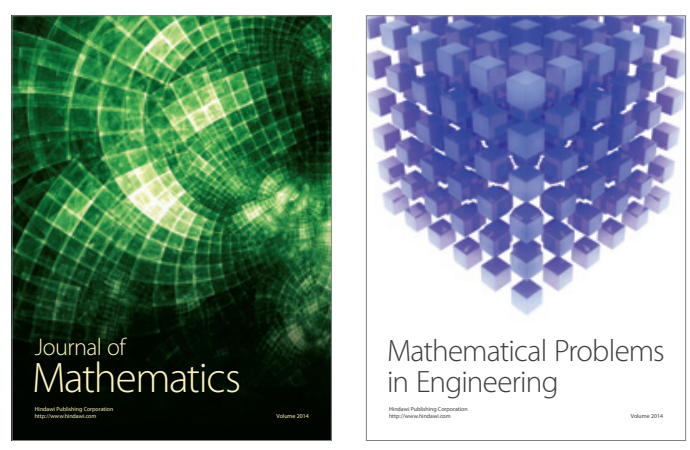

Mathematical Problems in Engineering
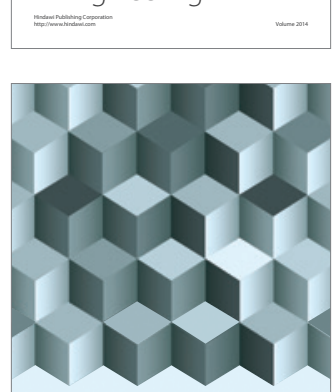

Journal of

Function Spaces
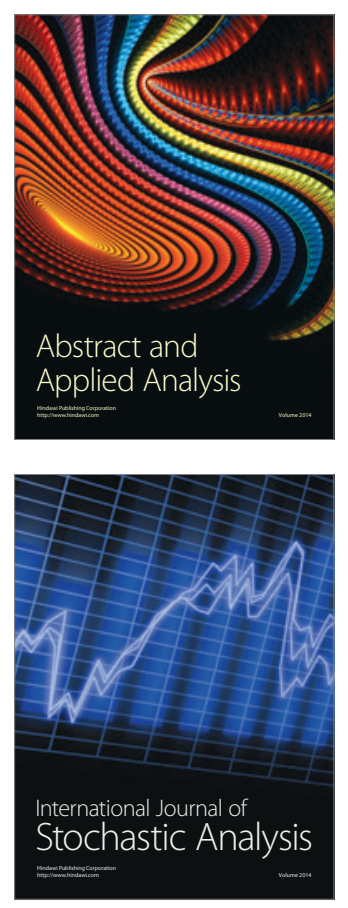

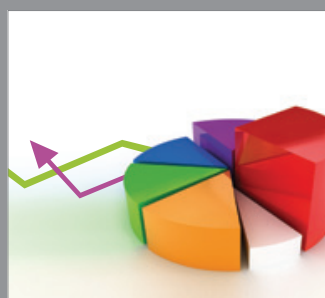

ournal of

Probability and Statistics

Promensencen
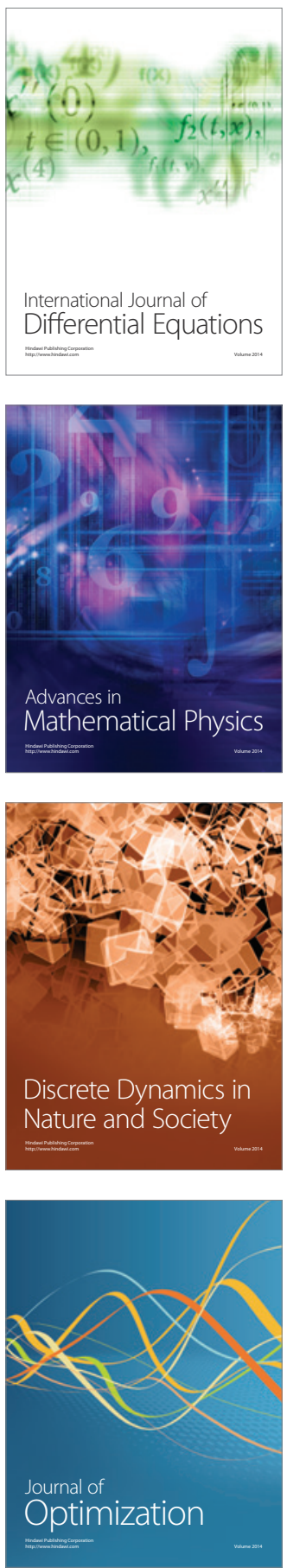